\title{
Leitfaden zur Meldepflicht
}

\section{Mirjam Mäusezahl}

Co-Leiterin Sektion Epidemiologie, Bundesamt für Gesundheit BAG

Mit dem revidierten Epidemiengesetz ist am 1. Januar 2016 auch die neue Verordnung über die Meldung von Beobachtungen übertragbarer Krankheiten des Menschen des Eidgenössischen Departements des Innern (kurz: Meldeverordnung des EDI) in Kraft getreten.

\section{Die wichtigsten Neuerungen der revidier- ten Meldeverordnung}

Die Verordnung erhöht die Transparenz betreffend der erhobenen Daten: Die Meldeinhalte sind mittels Oberbegriffen umschrieben und machen zusammen mit den zusätzlichen Angaben transparent, welche Daten erhoben werden und wohin diese weiterzuleiten sind. Die bewährte Zusammenarbeit zwischen der Ärzteschaft, den Laboratorien, den kantonsärztlichen Diensten und dem Bundesamt für Gesundheit (BAG) wird durch klar definierte Meldekriterien und vereinfachte Meldeprozesse gestärkt.

Bei einigen Beobachtungen wurde die Erhebung von Krankheits- bzw. Behandlungsverlauf eingeführt. Bei einigen Themen gab es zudem kleinere Anpassungenetwa bei den Meldefristen und den Angaben zur betroffenen Person -, mit dem Ziel, Ausbrüche frühzeitig erkennen und gezielt untersuchen zu können. Neu sind ab 1.1.2016 bzw. 5.3.2016 auch die Befunde zu Carbapenemase-bildenden Enterobacteriaceae bzw. zu ZikaFieber meldepflichtig. Die Verordnung ist auf der Internetseite des BAG in den drei Sprachen Deutsch, Französisch und Italienisch als PDF-Dokument zu finden (www.bag.admin.ch/epg).

\section{Leitfaden zur Meldepflicht und Poster mit meldepflichtigen Beobachtungen}

Damit die Anwendung der Meldeverordnung des EDI im Alltag leichter fällt, wurde ein Leitfaden zur Meldepflicht erarbeitet. Dieser soll den Meldepflichtigen ermöglichen, die Themen in alphabetischer Reihenfolge mit den dazugehörenden Meldekriterien, -fristen und -wegen rasch zu finden. Der Leitfaden steht als PDFDokument auf der Internetseite der Abteilung Übertragbare Krankheiten des BAG zur Verfügung (www. bag.admin.ch/infreporting).

Weiter ist ein grafisch ansprechendes Poster im Format A3 mit einem Überblick zu den meldepflichtigen übertragbaren Krankheiten als Bundespublikation bestellbar (www.bundespublikationen.admin.ch, Bestellnummer: 316.521.d). Beide Dokumente sind in Deutsch und Französisch verfügbar.

Rückmeldungen und Anregungen zum Leitfaden nehmen wir gerne entgegen (epi[at]bag.admin.ch). 